\title{
Determination of Duchenne muscular dystrophy carrier status by single strand conformation polymorphism analysis of deleted regions of the dystrophin locus
}

\author{
Robert I Richards, Kathryn Friend
}

\begin{abstract}
The molecular characterisation of the dystrophin gene, mutations in which are responsible for $X$ linked Duchenne and Becker muscular dystrophies, has led to an array of strategies for the diagnosis of affected subjects and carriers. Initially these were based on blotting and hybridisation technologies but have recently been largely superseded by PCR based techniques which afford greater speed and sensitivity. We describe the use of single strand conformation polymorphism to detect heterozygosity in regions of the dystrophin locus which are deleted in affected males, to determine the carrier status of their female relatives.
\end{abstract}

Duchenne (and Becker) muscular dystrophy is characterised by mutation of the dystrophin locus at $\mathrm{Xp} 21 .^{1}$ These mutations are frequently deletions ${ }^{2}$ which can be detected in 60 to $70 \%$ of affected males by Southern blot analysis. ${ }^{12}$ This technique also allows determination of carrier status of females in pedigrees affected by deletion either by dosage differences or detection of junction fragments. Pulsed field gel analysis of females at risk has been used to determine carrier status by detection of dystrophin sequences of altered mobility owing to deletion. ${ }^{3}$ The utility of blotting techniques is however being rapidly surpassed by polymerase chain reaction (PCR) based approaches. Chamberlain et al have described a multiplex PCR involving the simultaneous amplification of the deletion prone regions of the dystrophin locus which can detect $37 \%$ of affected males by the absence of PCR products.

Department of Cytogenetics and Molecular Genetics, Adelaide Children's Hospital, North Adelaide, South Australia 5006, Australia.

R I Richards, K Friend

Correspondence to Dr Richards.

Received for publication 15 April 1991.

Revised version accepted for publication 22 May 1991.
Recently, Beggs et al have characterised an additional nine PCRs, again in a single multiplex reaction, which in combination with an extended Chamberlain et $a l^{6}$ multiplex allows for detection of almost all males affected by deletion. While this analysis has dramatically improved the speed and accuracy of prenatal diagnosis in families where a deletion has been characterised, the carrier status of females in these affected pedigrees relies almost solely on linkage analysis. ${ }^{7}$ Linkage analysis has also benefited from PCR with the conversion of some RFLPs to PCR based assays ${ }^{89}$ and the characterisation of AC repeat microsatellite polymorphisms which flank the dystrophin gene. ${ }^{10-13}$ The utility and accuracy of linkage analysis are affected by the need for extended pedigree analysis to establish whether the pedigree represents a new mutation (about one third of all $\mathrm{D} / \mathrm{BMD}$ affected males represent new mutations) and also by the extraordinary size of the dystrophin gene which sees $12 \%$ recombination between its extremities. ${ }^{14}$ Females at risk for carrying a deletion can be shown to be either non-carriers or gonadal mosaics if they are heterozygous for a polymorphism within the deleted region. Only one Southern blot based RFLP marker, P20, is located in a deletion prone region, enabling determination of heterozygosity for potential carriers of this deletion. Carrier status determination by PCR dosage analysis has been problematical because of the nonlinearity of the reaction after about 19 cycles. While this has been successfully overcome to some extent by limiting PCR to 20 cycles and the use of sensitive spectrophotometric densitometry, technical problems and lack of reproducibility ${ }^{15}$ make this approach unsatisfactory for general use.

A simple and effective means of improving carrier detection in families with a PCR detectable deletion would be to identify polymorphisms in the deleted PCR multiplex band. This would allow heterozygous females to be identified as non-carriers or possibly gonadal mosaics depending upon their position in the pedigree. Sequence polymorphism resulting from nucleotide substitution is far more frequent than observed by RFLP analysis alone and 
several techniques have been devised to improve the detection of this type of polymorphism. The chemical cleavage method of Cotton et al, ${ }^{16}$ while effective, is technically demanding (when considered for routine diagnosis) and relies on characterisation of individual mutations. Orita et $a l^{17}$ have described a method which relies on the effect of nucleotide substitutions on the tertiary structure of single stranded DNA sequences and therefore their mobility in non-denaturing gel electrophoresis. This procedure has distinct advantages over the chemical cleavage method in that only simple physical manipulation of the samples is required before electrophoresis, and multiple nucleotide substitutions have the potential to be simultaneously detected as multiple alleles for the one DNA sequence, thus increasing the likelihood of heterozygosity. In this paper we describe the use of single stranded conformation polymorphisms (SSCP) to assign the carrier status of females in Duchenne muscular dystrophy pedigrees for whom a PCR deletion has been observed.

\section{Materials and methods}

Chromosomal DNA from affected family members was obtained from whole blood by the method of Wyman and White. ${ }^{18}$ Large kindred family DNA was obtained from the Centre d'Etude de Polymorphisme Humain (CEPH), Paris, France.

Multiplex PCR conditions were as described by Chamberlain et $a l^{4}$ except for the addition of $10 \mu \mathrm{Ci}$ of $\alpha-{ }^{32} \mathrm{PdCTP}$ to each $10 \mu \mathrm{l}$ reaction and the use of only two pairs of oligonucleotide primers for each of the multiplex reactions. Chromosomal DNA was isolated from whole blood from each member of the affected pedigrees. Products from the PCR were diluted 100 fold in $0.1 \%$ SDS, $10 \mathrm{mmol} / 1$ EDTA, followed by a 1:1 dilution in $95 \%$ formamide, $20 \mathrm{mmol} / 1$ EDTA, 0.05\% bromophenol blue, 0.05\% xylene cyanol. The samples were then heated to $90^{\circ} \mathrm{C}$ for three minutes and then loaded onto $4.5 \%$ non-denaturing acrylamide gels (49:1 ratio of acrylamide to bis-acrylamide) measuring $0.4 \mathrm{~mm} \times 20 \mathrm{~cm} \times 38 \mathrm{~cm}$ and containing $90 \mathrm{mmol} / 1$ Tris-borate (pH 8.3), $2 \mathrm{mmol} / 1 \mathrm{EDTA}$, and $10 \%$ glycerol. Electrophoresis was at 600 volts for 20 hours. Gels were dried under vacuum then autoradiographed at $-70^{\circ} \mathrm{C}$ with an intensifying screen.

In the reduced multiplex PCR used for SSCP analysis band $\mathbf{b}$ is the product generated across exon 17 of the dystrophin gene whereas band $e$ is the product generated across the exon contained in the $0.5 \mathrm{~kb}$ HindIII fragment detected with $\mathrm{cDNA}$ probe 7. This exon has been designated as exon 45 by Beggs et al. ${ }^{5}$

\section{Results}

Of 28 unrelated male probands affected by Duchenne or Becker muscular dystrophy and identified as having a deletion by multiplex PCR, 16 were deleted for band e. We therefore chose to concentrate on this region to evaluate SSCP analysis for carrier detection. While band $b$ was deleted in only three of the remaining affected males we chose to use it as an internal positive control in gel electrophoresis as it was easily resolved from the band e products. Simultaneous SSCP analysis of the entire multiplex PCR gave complex banding patterns which were difficult to interpret (data not shown). Simultaneous typing of bands $b$ and e gave simple, reproducible results (fig 1 ) which were easy to genotype. After denaturation and electrophoresis, the
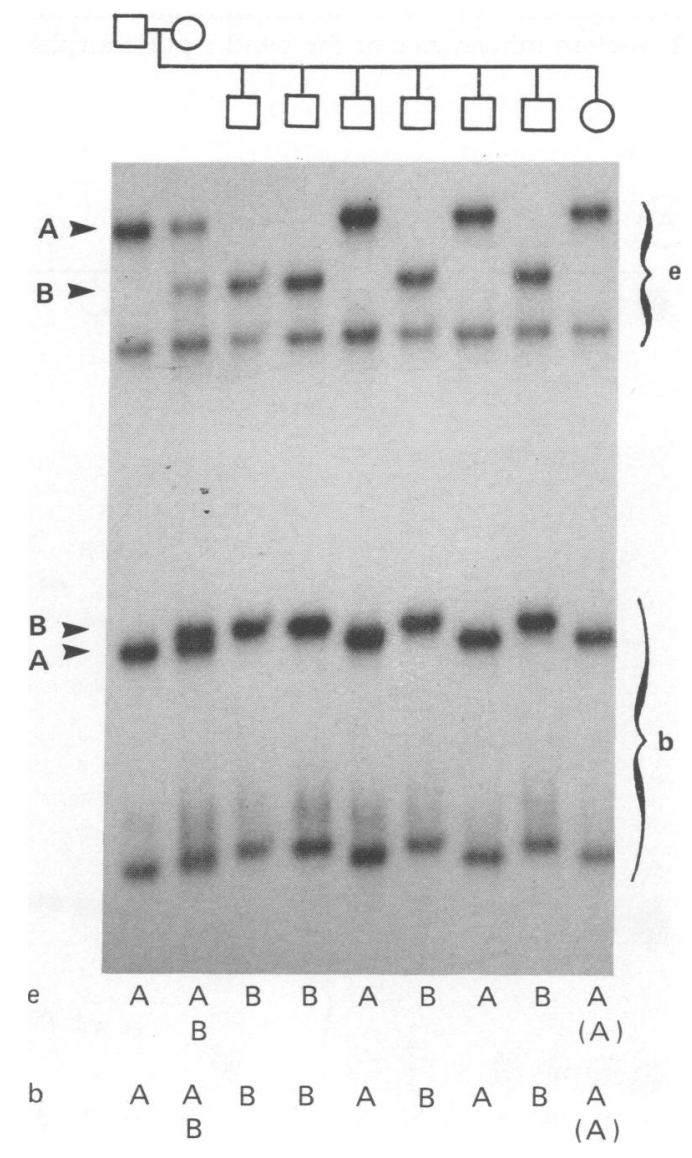

Figure 1 Codominant inheritance of SSCP markers at the dystrophin locus. This figure shows CEPH pedigree no 17 which was informative for SSCPs of both bands $e$ and $b$. Polymorphism of the upper strand of each band is indicated as either $A$ or $B$. Genotypes of each subject in the pedigree are given underneath the autoradiograph. Brackets indicate inferred genotype. 
two strands of DNA from each band are clearly resolved. Genotyping of unrelated CEPH and normal control subjects showed three common alleles at band $\mathrm{e}$ which were distinguished by alteration in the electrophoretic mobility of either or both of the single strands (figs 1 and 2). The 'upper' strand exhibited much greater alteration in mobility for the $B$ allele than its complementary 'lower' strand when compared to the more common A allele. For the $\mathrm{C}$ allele a greater shift in mobility was observed in the lower strand (fig 2B). The molecular basis for this is not known but probably reflects the different base composition of the two strands. Since G can pair with $T$ the conformations that the two strands adopt are unlikely to be mirror images.

The observed allele frequency for the band e polymorphism in 52 unrelated $\mathrm{X}$ chromosomes was eA (0.54), eB (0.42), and eC (0.04) with an observed heterozygosity of $53 \%$ in 22 unrelated females. Mendelian inheritance of the band e polymorphism was shown by genotyping of CEPH pedigrees (fig 1). Co-dominant segregation was observed.

Under the same gel electrophoresis conditions two alleles were observed for the dystrophin multiplex PCR products $b$ (fig 1 ) and $f$ (data not shown). In each case these were significantly less informative than band e. The observed allele frequencies for band $b$ in 24 unrelated $X$ chromosomes were bA $(0.93)$ and bB (0.17) with an observed heterozygosity of $28 \%$ in eight unrelated females, while for band $f$ allele frequencies in 34 unrelated $\mathrm{X}$ chromosomes were $\mathrm{fA}(0.88)$ and $\mathrm{fB}(0.12)$ with an observed heterozygosity of $21 \%$ in 16 unrelated females. No polymorphism was observed for the other dystrophin multiplex PCR products under the electrophoretic conditions used.

In order to determine the utility of SSCP analysis at the dystrophin locus several affected pedigrees where the proband was deleted for e were genotyped. Fig $2 \mathrm{~A}$ shows that the mother of an affected
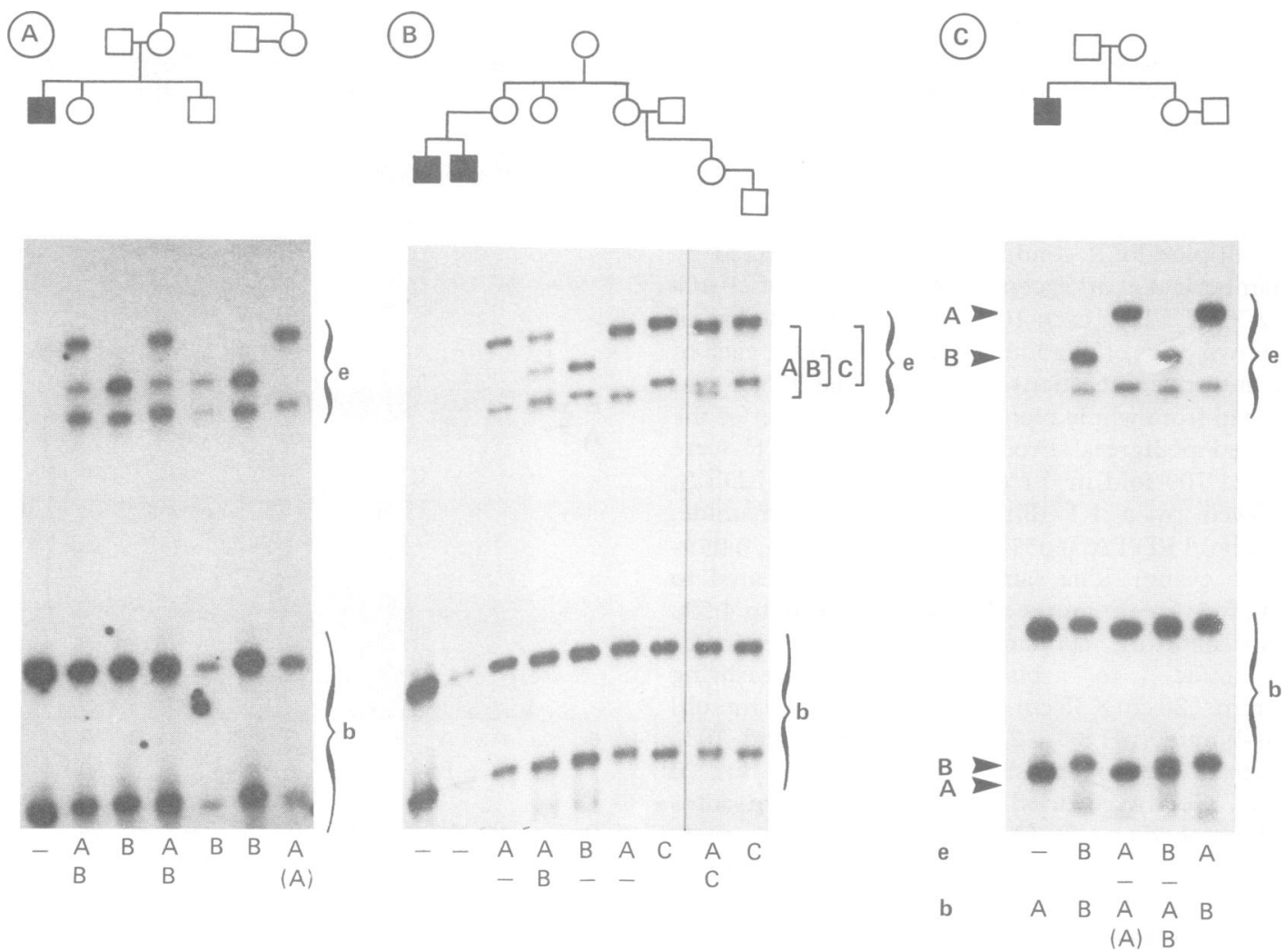

Figure 2 DMD affected pedigrees. SSCP analysis of three pedigrees with affected subjects deleted for band e. Genotypes are indicated below the respective lanes of the autoradiographs. Band $b$ was included as a positive control for the PCR and was only polymorphic in pedigree C. Brackets indicate inferred genotype. For pedigree B pairs of bands from each allele of the e PCR product are bracketed. 
boy deleted for e is heterozygous for the deleted region and therefore is either not a carrier or is a gonadal mosaic. The proband's sister is also heterozygous for the deleted region and is therefore not a carrier and does not need to consider prenatal diagnosis. Fig 2B shows a second pedigree in which affected boys are deleted for e. In this case, the female at the head of the pedigree can be clearly shown to carry the deletion, since she exhibits only the $\mathrm{eB}$ allele while two of her daughters carry only the eA allele. All three of these women are therefore hemizygous for this region of the $\mathrm{X}$ chromosome and are therefore carriers of the deletion. While one of the carrier daughters has passed on the deleted $\mathrm{X}$ chromosome to her two sons, the other carrier daughter has not passed the deleted $\mathrm{X}$ chromosome to her daughter. Fig 2C shows another pedigree where the female at the head of the pedigree was found to carry the deletion. In this case the deleted $\mathrm{X}$ chromosome has been inherited by the proband's sister who is thereby shown to be a carrier. This family is also informative for the band b SSCP.

\section{Discussion}

We describe the application of SSCP analysis to carrier detection in pedigrees known to have subjects affected with Becker or Duchenne muscular dystrophy deleted at the dystrophin locus. We optimised the conditions for observing this type of polymorphism at the most commonly deleted region, corresponding to band e. Polymorphism observed at band $b$ and $f$ suggests that this approach will be generally applicable to carrier detection in informative families. Variation of electrophoretic conditions $^{17}$ should enable additional polymorphisms to be observed in other dystrophin PCR products and further alleles may be detected in band e.

The polymorphic nature of band e suggests that it, and perhaps all of the dystrophin multiplex bands, have the potential for use as polymorphic markers for linkage analysis in affected pedigrees in which no deletion can be detected.

The successful application of SSCP markers to Duchenne/Becker muscular dystrophy carrier detection suggests that they may be an important new source of markers for diagnosis of genetic disease.

The authors wish to thank Dr Takao Sekiya for communicating the PCR-SCCP method before publication and Drs Grant Sutherland, Eric Haan, and John Mulley for discussions and review of this manuscript. Robert Richards wishes to thank Shelley Richards for support and encouragement during the course of these studies. This work was supported by a grant from the Adelaide Children's Hospital Research Trust.

1 Koenig M, Hoffman EP, Bertelson CJ, Monaco AP, Feener C, Kunkel LM. Complete cloning of the Duchenne muscular dystrophy (DMD) CDNA and preliminary genomic organization of the DMD gene in normal and affected individuals. Cell 1987;50:509-17.

2 Koenig M, Beggs AH, Moyer M, et al. The molecular basis for Duchenne versus Becker muscular dystrophy: correlation of severity with type of deletion. Am ₹ Hum Genet 1989;45:498506.

3 Den Dunnen JT, Bakker E, Klein Breteler EG, Pearson PL, van Ommen GJB. Direct detection of more than $50 \%$ of the Duchenne muscular dystrophy mutations by field inversion gels. Nature 1987;329:640-2.

4 Chamberlain JS, Gibbs RA, Ranier JE, Nguyen PN, Caskey CT. Deletion screening of the Duchenne muscular dystrophy locus via multiplex DNA amplification. Nucleic Acids Res 1988;16:11141-56.

5 Beggs AH, Koenig M, Boyce FM, Kunkel LM. Detection of $98 \%$ of DMD/BMD gene deletions by polymerase chain reaction. Hum Genet 1990;86:45-8.

6 Chamberlain JS, Gibbs RA, Ranier JE, Caskey CT. Multiplex PCR for the diagnosis of Duchenne muscular dystrophy. In: Innis MA, Gelfand DH, Sninsky JJ, White TJ, eds. PCR protocols: a guide to methods and applications. New York: Academic Press, 1990: 272-81.

7 Prior TW, Blasco PA, Dove JL, Leshner RT, Gruemer HD. Use of DNA probes in detecting carriers of Duchenne muscular dystrophy: selected case studies. Clin Chem 1989; 35:679-83.

8 Roberts RG, Cole CG, Hart KA, Bobrow M, Bentley DR. Rapid carrier and prenatal diagnosis of Duchenne and Becker muscular dystrophy. Nucleic Acids Res 1989;17:811.

9 Roberts RG, Bobrow M, Bentley DR. A MaeIII polymorphism near the dystrophin gene promoter by restriction of amplified DNA. Nucleic Acids Res 1990;18:1315.

10 Oudet C, Heilig R, Mandel JL. An informative polymorphism detected by PCR at the $3^{\prime}$ end of the dystrophin gene. Hum Genet 1990;84:283-5.

11 Beggs AH, Kunkel LM. A polymorphic CACA repeat in the 3 untranslated region of dystrophin. Nucleic Acids Res 1990; 18:1931.

12 Powell JF, Fodor FH, Cockburn DJ, Monaco AP, Craig IW. A dinucleotide repeat polymorphism at the DMD locus. Nucleic Acids Res 1991;19:1159.

13 Feener CA, Boyce FM, Kunkel LM. Rapid detection of CA polymorphisms in cloned DNA: application to the $5^{\prime}$ region of the dystrophin gene. Am f Hum Genet 1991;48:621-7.

14 Abbs S, Roberts RG, Mathew CG, Bentley DR, Bobrow M. Accurate assessment of intragenic recombination frequency within the Duchenne muscular dystrophy gene. Genomics 1990;7:602-6.

15 Speer A, Spiegler AWJ, Hanke R, et al. Possibilities and limitation of prenatal diagnosis and carrier determination for Duchenne and Becker muscular dystrophy using cDNA probes. F Med Genet 1989;26:1-5.

16 Cotton RGH, Rodrigues NR, Campbell RD. Reactivity of cytosine and thymine in single-base pair mismatches with hydroxylamine and osmium tetroxide and its application to the study of mutations. Proc Natl Acad Sci USA 1988; 85:4397-401.

17 Orita $M$, Iwahana $H$, Kanazawa $H$, Hayashi $K$, Sekiya $T$. Detection of polymorphisms of human DNA by gel electrophoresis as single-strand conformation polymorphisms. Proc Natl Acad Sci USA 1989;86:2766-70.

18 Wyman AR, White R. A highly polymorphic locus in human DNA. Proc Natl Acad Sci USA 1980;77:6754-8. 